\title{
A study to evaluate the relationship between socio-economic, demographic characteristics and complications of acceptors in double puncture laparoscopic and conventional tubal ligation procedures
}

\author{
Sudhir Babu Palli ${ }^{1} *$, Vijaya Lakshmi Akkupalli ${ }^{2}$
}

\author{
${ }^{1}$ Department of Obstetrics \& Gynecology, Viswabharathi Medical College and General Hospital, Kurnool, Andhra \\ Pradesh, India \\ ${ }^{2}$ Department of Physiology, Viswabharathi Medical College and General Hospital, Kurnool, Andhra Pradesh, India
}

Received: 27 January 2013

Accepted: 30 January 2013

\section{*Correspondence:}

Dr. Sudhir Babu Palli

E-mail: magadhira@gmail.com

\begin{abstract}
Background: To discover the relationship between the socio-economic, demographic characteristics and complications for acceptors following Double puncture laparoscopic (DPL ) and conventional tubal ligation (CTL) procedures in rural and urban communities and additionally, we also sought to study the occurrence of post tubal ligation syndrome.

Methods: A follow up study on 1000 women over a period of two years was conducted. The study was divided into 2 groups: 500 women undergone double puncture laparoscopic (DPL) sterilization and another 500 women underwent conventional tubal ligation (CTL). The acceptors were personally interviewed as per the guide lines in the proforma in the field at their door steps with help of multipurpose health workers (ANM) of the area concerned in the rural areas.

Results: The mean age of DPL and CTL groups were 24.4 years and 23.8 years, respectively. The acceptance of type of procedure was almost equal in all religious groups. As the level of education increases there is clear correlation with DPL acceptance. The overall acceptance of tubectomy was higher in house wives (61\%) than the working women $(39 \%)$. However house wives preferred to undergone conventional tubectomy more $(63 \%)$ than the DPL (59\%). The DPL acceptors were more of higher economic status (49\%) whereas CTL acceptors were more of lower economic status (54.4\%). The overall complications of DPL acceptors were lower than CTL acceptors. Moreover, these complications didn't influence with socio-economic, demographic characteristics.

Conclusions: Age, religion, literacy status, economic status \& occupation of acceptors didn't influence the occurrence of complications and post tubal ligation syndrome does exist in both groups.
\end{abstract}

Keywords: Socio-economic status, DPL, Post tubal ligation syndrome

\section{INTRODUCTION}

The most serious problem India is facing today is the rapid growth of its population. The current population of India in 2012 is estimated to be 1.22 billion. Over populated countries like India depend mainly on sterilization to control population explosion. Female sterilization is a relatively simple procedure that involves permanently blocking the fallopian tubes to prevent fertilization. The procedure was first used in the early 19th century by James Blundell, and the first published report of this procedure was in 1881. By the mid-20th century, female sterilization had begun to gain popularity. Many modifications and new techniques have been developed since, to improve effectiveness, safety, and reversibility. Today, greatly simplified procedures performed under local anesthesia and in ambulatory settings have helped minimize the complications associated with general anesthesia and have permitted the expansion of services to lower levels of the health service system in many countries. Serious complications are rare and occur in fewer than $2 \%$ of all female sterilization procedures. ${ }^{1,2}$

The development of laparoscopy and minilaparotomy has made the procedure readily available even in developing countries. Complications are rare and occur in fewer than $1 \%$ of all female sterilization procedures (Stewart \& Carignan, 1998). ${ }^{3}$ The World Health Organization 
(WHO) definition for complications following female sterilization is: "problems directly related to the surgery or the anesthesia that occur within 42 days and that require intervention and management beyond what would be normally provided." Examples include infection, bleeding, unintended injury to internal organs, and depressed respiration or blood pressure due to anesthesia (WHO, 1992). ${ }^{4}$

Complications can be categorized as minor or major. Major complications require unintended hospitalization or surgery, blood transfusion, or treatment of lifethreatening events or events that result in death (WHO, 1992). ${ }^{4}$ Minor complications are those that require intervention and management beyond what would normally be provided, but do not progress to any of the five events mentioned above. ${ }^{1,4}$ One of the delayed complication is post tubal sterilization syndrome, it consists of irregular menstrual cycles, dysmenorrhea, menorrhagia, and midcycle bleeding. Some scientists speculate that interference with the utero-ovarian blood supply and subsequent disturbance of ovarian function bring about post tubal ligation syndrome changes. ${ }^{5}$

There are limited studies on the assessment of socioeconomic, demographic characteristics with relation to complications following the double puncture laparoscopic (DPL) and conventional tubal ligation (CTL) procedures. So the objective of this study was to find out relationship between socio-economic, demographic characteristics and complications for acceptors following the double puncture laparoscopic (DPL) \& conventional tubal ligation (CTL) sterilization procedures and also to evaluate the occurrence of post tubal ligation syndrome.

\section{METHODS}

A 2 years follow-up prospective study of laparoscopic sterilization (DPL) and conventional tubectomy (CTL) in Family Planning Unit, Government Maternity hospital, Tirupati, A.P., India was undertaken (January 2001March 2003). 500 voluntary conventional tubectomy accepters and 500 Voluntary DPL acceptors are selected and operated. The selection criteria was strictly followed, so as not to interfere with the interpretation of results as most of the morbidity or complications could arise because of preexisting conditions like MTP, Hysterotomy, LSCS, menstrual disorders etc. The cases were personally attended during the procedure and followed up post operatively and complications noted as per the guidelines in the proforma.

All cases were personally interviewed at their door steps with the guidance of multipurpose health workers of the area concerned in the rural areas and in urban areas with the help of ANM's welfare workers of post-partum unit in Government Maternity Hospital, Tirupati, AP, India.
Questionnaires were originally prepared in English and were translated into local language. The interviewers' selected were ANMs of post partum unit and were trained to the level of satisfaction and allowed to conduct the survey. Collected data were statistically analyzed by chisquare test.

\section{RESULTS}

The mean age of acceptors in DPL group was 24.4 \& conventional tubectomy group was 23.8. The overall mean age of both groups was 24.1 years (Table 1 ).

In DPL acceptors, Hindus, Muslims and Christians were $87.80 \%, 7.80 \%$ and $4.40 \%$, respectively. Whereas in CTL acceptors, Hindus, Muslims and Christians were $87.60 \%$, $7.20 \%$, and $4.40 \%$, respectively. The religion wise distribution was found to be almost equal in both groups (Table 2).

As per Table 3, literacy status was below primary education in conventional tubectomy acceptors, which was more than DPL (78.80\%). But literacy status was above secondary education in DPL selected women, which more than conventional tubectomy $(40.80 \%)$.

House wives preferred the conventional tubectomy procedure $(63 \%)$ while the working women preferred DPL (41\%) (Table 4).

As evident from table 5, The DPL acceptors were more from higher economic status group (4.9\%) while conventional tubectomy acceptors were of lower economic status group $(54.40 \%)$.

With reference to complications, only 2 patients required hospital admission, both were of ectopic pregnancy. The major morbidity for both groups was $0.2 \%$ and was almost equal. Most of the complications were evenly placed with little difference in different age groups. The acceptors above 30 years had specific complications like PID, Dyspareunia \& scar tenderness and these complications were more seen in conventional tubectomy group. The other complications were comparatively same in both acceptors in all age groups (Table 6 and 7).

Most of the complications were evenly observed in all groups. PID \& Dyspareunia seemed to be more in Muslim acceptors (Table 8 and 9).

Most of the complications with reference to literacy status were evenly seen in both groups. Specific complications like PID and Dyspareunia were more in acceptors whose education was below primary level (Table 10 and 11). 
Table 1: Distribution of DPL and conventional tubectomy acceptors according to age.

\begin{tabular}{|c|c|c|c|c|c|c|}
\hline \multirow[t]{2}{*}{ Age } & \multicolumn{2}{|c|}{ DPL } & \multicolumn{2}{|c|}{ CTL } & \multicolumn{2}{|c|}{ Total } \\
\hline & $\begin{array}{l}\text { No. of } \\
\text { Cases }\end{array}$ & $\%$ & $\begin{array}{l}\text { No. of } \\
\text { Cases }\end{array}$ & $\%$ & No. of Cases & $\%$ \\
\hline Below 20 Years & 64 & 12.80 & 71 & 14.20 & 135 & 13.50 \\
\hline $21-25$ Years & 286 & 57.20 & 265 & 53.00 & 551 & 55.10 \\
\hline $26-30$ Years & 131 & 26.20 & 140 & 28.00 & 271 & 27.10 \\
\hline $30-35$ Years & 16 & 3.20 & 20 & 4.00 & 36 & 3.60 \\
\hline $35-40$ Years & 3 & 0.60 & 4 & 0.80 & 7 & 0.70 \\
\hline Total No. of Cases \& \% & 500 & 100 & 500 & 100 & 1000 & 100 \\
\hline
\end{tabular}

Table 2: Distribution of DPL and CTL acceptors according to religion.

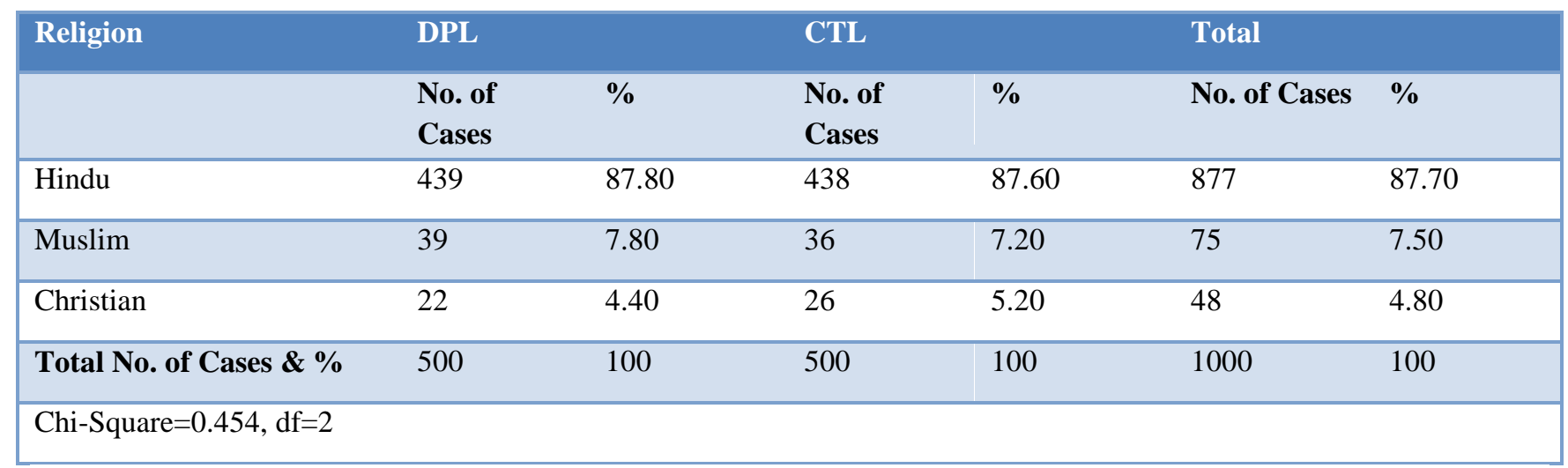

Table 3: Distribution of DPL and conventional tubectomy acceptors literacy status.

\begin{tabular}{|c|c|c|c|c|c|c|}
\hline \multirow[t]{2}{*}{ Education } & \multicolumn{2}{|c|}{ DPL } & \multicolumn{2}{|c|}{ CTL } & \multicolumn{2}{|c|}{ Total } \\
\hline & $\begin{array}{l}\text { No. of } \\
\text { Cases }\end{array}$ & $\%$ & $\begin{array}{l}\text { No. of } \\
\text { Cases }\end{array}$ & $\%$ & No. of Cases & $\%$ \\
\hline Illiterate & 92 & 18.40 & 208 & 41.60 & 300 & 30.00 \\
\hline Primary & 204 & 40.80 & 186 & 37.20 & 390 & 39.00 \\
\hline Secondary & 120 & 24.00 & 93 & 18.60 & 213 & 21.30 \\
\hline Inter and Above & 84 & 16.80 & 13 & 2.60 & 97 & 9.70 \\
\hline $35-40$ Years & 3 & 0.60 & 4 & 0.80 & 7 & 0.70 \\
\hline Total No. of Cases \& \% & 500 & 100 & 500 & 100 & 1000 & 100 \\
\hline Chi-Square $=101.076, \mathrm{df}=3$ & & & & & & \\
\hline
\end{tabular}

Table 4: Distribution of DPL and conventional tubectomy acceptors occupation-wise.

\begin{tabular}{|c|c|c|c|c|c|c|}
\hline \multirow[t]{2}{*}{ Occupation } & \multicolumn{2}{|c|}{ DPL } & \multicolumn{2}{|c|}{ Con Tubectomy } & \multicolumn{2}{|c|}{ Total } \\
\hline & $\begin{array}{l}\text { No. of } \\
\text { Cases }\end{array}$ & $\%$ & $\begin{array}{l}\text { No. of } \\
\text { Cases }\end{array}$ & $\%$ & No. of Cases & $\%$ \\
\hline House wives & 295 & 59.00 & 315 & 63.00 & 610 & 61.00 \\
\hline Working Women & 205 & 41.00 & 185 & 37.00 & 390 & 39.00 \\
\hline Total No. of Cases \& \% & 500 & 100 & 500 & 100 & 1000 & 100 \\
\hline
\end{tabular}


Table 5: Distribution of DPL and conventional tubectomy acceptors regarding economic status.

\begin{tabular}{|lllllll|}
\hline Economic Status & DPL & \multicolumn{3}{l}{ Con Tubectomy } & \multicolumn{2}{l|}{ Total } \\
& $\begin{array}{l}\text { No. of } \\
\text { Cases }\end{array}$ & \% & $\begin{array}{l}\text { No. of } \\
\text { Cases }\end{array}$ & \% & No. Cases & \% \\
\hline Below 12000 Rs & 180 & 36.00 & 272 & 54.40 & 452 & 45.20 \\
\hline 12000-36000 Rs & 245 & 49.00 & 173 & 34.60 & 418 & 41.80 \\
\hline Above 36000 Rs & 75 & 15.00 & 55 & 11.00 & 130 & 13.00 \\
\hline Total No. of Cases \& \% & 500 & 100 & 500 & 100 & 1000 & 100 \\
\hline Chi-Square=34.204, df=2 & & & & & & \\
\hline
\end{tabular}

Table 6: Complications of CTL according to age.

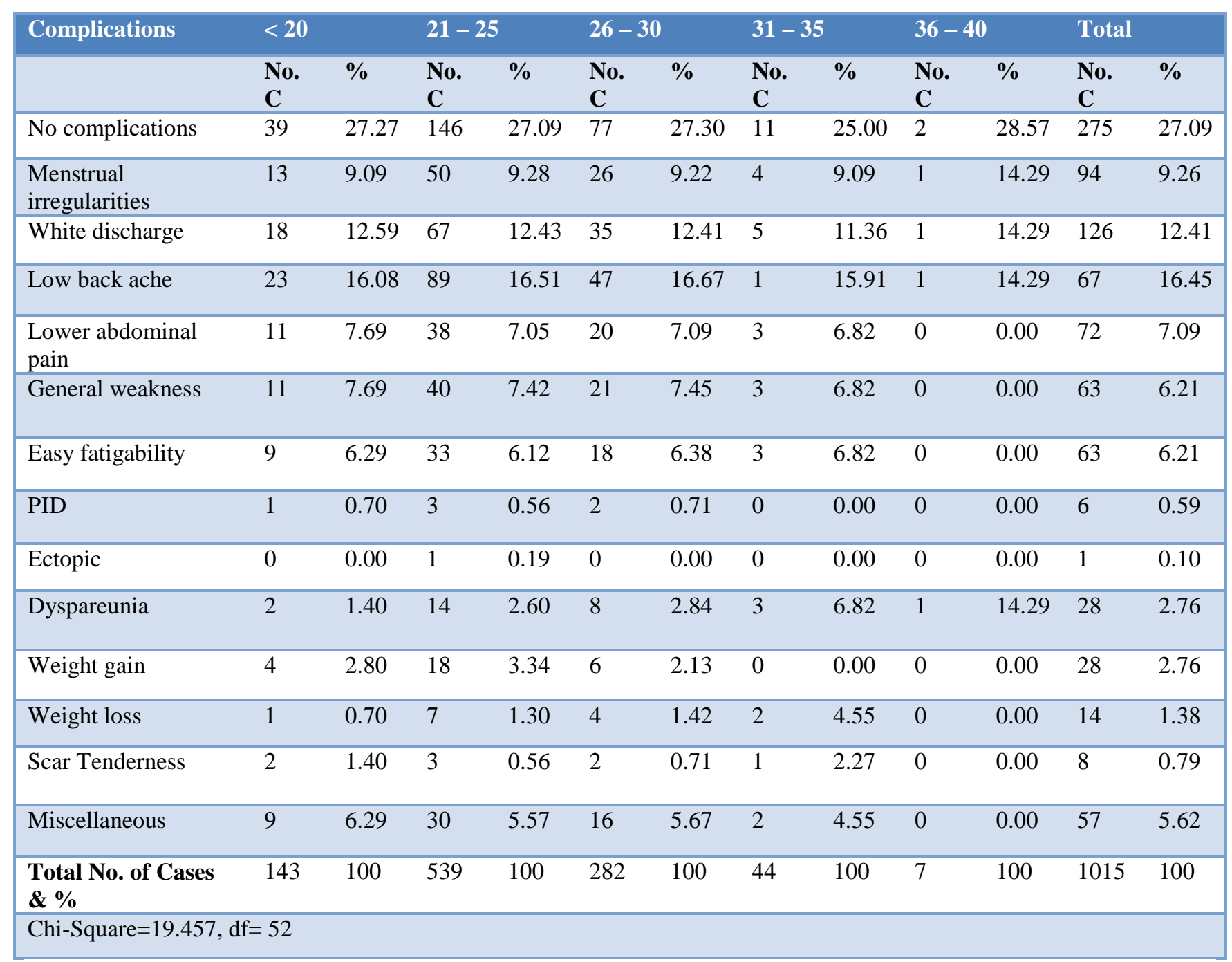

Table 7: Complications of DPL according to age.

\begin{tabular}{|c|c|c|c|c|c|c|c|c|c|c|c|c|}
\hline \multirow[t]{2}{*}{ Complications } & \multicolumn{2}{|c|}{$<20$} & \multicolumn{2}{|c|}{$21-25$} & \multicolumn{2}{|c|}{$26-30$} & \multicolumn{2}{|c|}{$31-35$} & \multicolumn{2}{|c|}{$36-40$} & \multicolumn{2}{|c|}{ Total } \\
\hline & $\begin{array}{l}\text { No. } \\
\text { C }\end{array}$ & $\%$ & $\begin{array}{l}\text { No. } \\
\text { C }\end{array}$ & $\%$ & $\begin{array}{l}\text { No. } \\
\text { C }\end{array}$ & $\%$ & $\begin{array}{l}\text { No. } \\
\text { C }\end{array}$ & $\%$ & $\begin{array}{l}\text { No. } \\
\text { C }\end{array}$ & $\%$ & $\begin{array}{l}\text { No. } \\
\text { C }\end{array}$ & $\%$ \\
\hline No complications & 42 & 37.50 & 191 & 38.43 & 86 & 36.91 & 10 & 32.26 & 1 & 10.00 & 330 & 37.37 \\
\hline
\end{tabular}




\begin{tabular}{|lllllllllllll|}
\hline $\begin{array}{l}\text { Menstrual } \\
\text { Irregularities }\end{array}$ & 9 & 8.04 & 38 & 7.65 & 17 & 7.30 & 2 & 6.45 & 0 & 0.00 & 66 & 7.47 \\
\hline White discharge & 17 & 15.18 & 73 & 14.69 & 34 & 14.59 & 4 & 12.90 & 0 & 0.00 & 128 & 14.50 \\
\hline Low back ache & 16 & 14.29 & 70 & 14.08 & 32 & 13.73 & 3 & 9.68 & 1 & 10.00 & 122 & 13.82 \\
\hline Lower abdominal pain & 5 & 4.46 & 4 & 4.83 & 12 & 5.15 & 1 & 3.23 & 0 & 0.00 & 48 & 5.44 \\
\hline General weakness & 6 & 5.36 & 27 & 5.43 & 14 & 6.01 & 1 & 3.23 & 0 & 0.00 & 48 & 5.44 \\
\hline Easy fatigability & 6 & 5.36 & 29 & 5.84 & 13 & 5.58 & 2 & 6.45 & 2 & 20.00 & 52 & 5.89 \\
\hline PID & 0 & 0.00 & 1 & 0.20 & 0 & 0.00 & 0 & 0.00 & 0 & 0.00 & 4 & 0.45 \\
\hline Ectopic & 0 & 0.00 & 1 & 0.20 & 0 & 0.00 & 0 & 0.00 & 0 & 0.00 & 1 & 0.11 \\
\hline Dyspareunia & 0 & 0.00 & 11 & 2.21 & 6 & 2.58 & 2 & 6.45 & 1 & 10.00 & 20 & 2.27 \\
\hline Weight gain & 2 & 1.79 & 12 & 2.41 & 4 & 1.72 & 1 & 3.23 & 0 & 0.00 & 19 & 2.15 \\
\hline Weight loss & 1 & 0.89 & 8 & 1.61 & 4 & 1.72 & 1 & 3.23 & 0 & 0.00 & 14 & 1.59 \\
\hline Scar Tenderness & 0 & 0.00 & 0 & 0.00 & 0 & 0.00 & 0 & 0.00 & 0 & 0.00 & 0 & 0.00 \\
\hline Miscellaneous & 8 & 7.14 & 11 & 2.21 & 9 & 3.86 & 4 & 12.90 & 5 & 50.00 & 37 & 4.19 \\
\hline $\begin{array}{l}\text { Total No. of Cases \& } \\
\text { \% } 112\end{array}$ & 100 & 497 & 100 & 233 & 100 & 31 & 100 & 10 & 100 & 883 & 100 \\
\hline Chi-Square=85.141, df= 52 & & & & & & & & & & \\
\hline
\end{tabular}

Table 8: Complications of CTL according to religion.

\begin{tabular}{|lllllllll|}
\hline & Hindu & \multicolumn{3}{c}{ Christians } & \multicolumn{3}{c|}{ Muslims } \\
\hline & No. C & \% & No. C & \% & No. C & \% & No. C & \% \\
\hline No complications & 241 & 27.26 & 14 & 25.45 & 20 & 26.67 & 275 & 27.12 \\
\hline Menstrual irregularities & 82 & 9.28 & 5 & 9.09 & 7 & 9.33 & 94 & 9.27 \\
\hline White discharge & 110 & 12.44 & 7 & 12.73 & 9 & 12.00 & 126 & 12.43 \\
\hline Low back ache & 146 & 6.52 & 9 & 16.36 & 12 & 16.00 & 167 & 16.47 \\
\hline Lower abdominal pain & 63 & 7.13 & 4 & 7.27 & 5 & 6.67 & 72 & 7.10 \\
\hline General weakness & 67 & 7.58 & 4 & 7.27 & 5 & 6.67 & 76 & 7.50 \\
\hline Easy fatigability & 55 & 6.22 & 3 & 5.45 & 5 & 6.67 & 63 & 6.21 \\
\hline PID & 4 & 0.45 & 1 & 1.82 & 1 & 1.33 & 6 & 0.59 \\
\hline Ectopic & 1 & 0.11 & 0 & 0.00 & 0 & 0.00 & 1 & 0.10 \\
\hline Dyspareunia & 23 & 2.60 & 3 & 5.45 & 3 & 4.00 & 29 & 2.86 \\
\hline Weight gain & 21 & 2.38 & 1 & 1.82 & 1 & 1.33 & 23 & 2.27 \\
\hline Weight loss & 14 & 1.58 & 1 & 1.82 & 1 & 1.33 & 16 & 1.58 \\
\hline Scar Tenderness & 7 & 0.79 & 0 & 0.00 & 2 & 2.67 & 9 & 0.89 \\
\hline Miscellaneous & 50 & 5.66 & 3 & 5.45 & 4 & 5.33 & 57 & 5.62 \\
\hline Total No. of Cases \& \% & 884 & 100 & 55 & 100 & 75 & 100 & 1014 & 100 \\
\hline Chi-Square=8.352, df= 26 & & & & & & & & \\
\hline
\end{tabular}


Table 9: Complications of DPL according to religion.

\begin{tabular}{|c|c|c|c|c|c|c|c|c|}
\hline \multirow[t]{2}{*}{ Complications } & \multicolumn{2}{|l|}{ Hindu } & \multicolumn{2}{|c|}{ Christians } & \multicolumn{2}{|c|}{ Muslims } & \multicolumn{2}{|l|}{ Total } \\
\hline & No. C & $\%$ & No. C & $\%$ & No. C & $\%$ & No. C & $\%$ \\
\hline No complications & 290 & 37.71 & 16 & 38.10 & 24 & 33.33 & 330 & 37.37 \\
\hline Menstrual irregularities & 58 & 7.54 & 3 & 7.14 & 5 & 6.94 & 66 & 7.47 \\
\hline White discharge & 112 & 14.56 & 6 & 14.29 & 10 & 13.89 & 128 & 14.50 \\
\hline Low back ache & 108 & 14.04 & 5 & 11.90 & 9 & 12.50 & 122 & 13.82 \\
\hline Lower abdominal pain & 37 & 4.81 & 2 & 4.76 & 3 & 4.17 & 42 & 4.76 \\
\hline General weakness & 42 & 5.46 & 2 & 4.76 & 3 & 4.17 & 42 & 4.76 \\
\hline Easy fatigability & 46 & 5.98 & 2 & 4.76 & 4 & 5.56 & 52 & 5.89 \\
\hline PID & 3 & 0.39 & 0 & 0.00 & 1 & 1.39 & 4 & 0.45 \\
\hline Ectopic & 1 & 0.13 & 0 & 0.00 & 0 & 0.00 & 1 & 0.11 \\
\hline Dyspareunia & 3 & 1.69 & 2 & 4.76 & 5 & 6.94 & 20 & 2.27 \\
\hline Weight gain & 15 & 1.95 & 2 & 4.76 & 2 & 2.78 & 19 & 2.15 \\
\hline Weight loss & 11 & 1.43 & 1 & 2.38 & 2 & 2.78 & 14 & 1.59 \\
\hline Scar Tenderness & 0 & 0.00 & 0 & 0.00 & 0 & 0.00 & 0 & 0.00 \\
\hline Miscellaneous & 33 & 4.29 & 1 & 2.38 & 3 & 4.17 & 37 & 4.19 \\
\hline Total No. of Cases \& \% & 769 & 100 & 42 & 100 & 72 & 100 & 883 & 100 \\
\hline
\end{tabular}

Table 10: Complications of CTL according to literacy status.

\begin{tabular}{|lllllllllll|}
\hline Complications & Illiterate & \multicolumn{2}{c}{ Primary } & \multicolumn{2}{c}{ Secondary } & Inter \& Above & Total \\
\hline & No. C & \% & No. C & \% & No. C & \% & No. C & \% & No. C & \% \\
\hline No complications & 114 & 27.14 & 102 & 26.91 & 51 & 27.57 & 8 & 26.67 & 275 & 27.12 \\
\hline $\begin{array}{l}\text { Menstrual } \\
\text { irregularities }\end{array}$ & 39 & 9.29 & 35 & 9.23 & 17 & 9.19 & 3 & 10.00 & 94 & 9.27 \\
\hline White discharge & 52 & 12.38 & 47 & 12.40 & 23 & 12.43 & 4 & 13.33 & 126 & 12.43 \\
\hline Low back ache & 69 & 16.43 & 63 & 16.62 & 31 & 16.76 & 4 & 13.33 & 167 & 16.47 \\
\hline $\begin{array}{l}\text { Lower abdominal } \\
\text { pain }\end{array}$ & 30 & 7.14 & 27 & 7.12 & 13 & 7.03 & 2 & 6.67 & 72 & 7.10 \\
\hline General weakness & 32 & 7.62 & 28 & 7.39 & 14 & 7.57 & 2 & 6.67 & 76 & 7.50 \\
\hline Easy fatigability & 26 & 6.19 & 23 & 6.07 & 12 & 6.49 & 2 & 6.67 & 63 & 6.21 \\
\hline PID & 3 & 0.71 & 2 & 0.53 & 1 & 0.54 & 0 & 0.00 & 6 & 0.59 \\
\hline Ectopic & 1 & 0.24 & 0 & 0.00 & 0 & 0.00 & 0 & 0.00 & 1 & 0.10 \\
\hline Dyspareunia & 9 & 2.14 & 11 & 2.90 & 7 & 3.78 & 2 & 6.67 & 29 & 2.86 \\
\hline Weight gain & 11 & 2.62 & 10 & 2.64 & 1 & 0.54 & 1 & 3.33 & 23 & 2.27 \\
\hline
\end{tabular}




\begin{tabular}{|lcccccccccc|}
\hline Weight loss & 8 & 1.90 & 6 & 1.58 & 1 & 0.54 & 1 & 3.33 & 16 & 1.58 \\
\hline Scar Tenderness & 2 & 0.48 & 3 & 0.79 & 3 & 1.62 & 1 & 3.33 & 9 & 0.89 \\
\hline Miscellaneous & 24 & 5.71 & 22 & 5.80 & 11 & 5.95 & 0 & 0.00 & 57 & 5.62 \\
\hline $\begin{array}{l}\text { Total No. of Cases } \\
\text { \& \% }\end{array}$ & 420 & 100 & 379 & 100 & 185 & 100 & 30 & 100 & 1014 & 100 \\
\hline $\begin{array}{l}\text { Chi-Square=15.827, } \mathrm{df}=39 \\
\end{array}$ & & & & & & & & & \\
\hline
\end{tabular}

Table 11: Complications of DPL according to literacy status.

\begin{tabular}{|c|c|c|c|c|c|c|c|c|c|c|}
\hline \multirow[t]{2}{*}{ Complications } & \multicolumn{2}{|c|}{ Illiterate } & \multicolumn{2}{|c|}{ Primary } & \multicolumn{2}{|c|}{ Secondary } & \multicolumn{2}{|c|}{ Intermediate $\&$} & \multicolumn{2}{|l|}{ Total } \\
\hline & No. C & $\%$ & No. $\mathrm{C}$ & $\%$ & No. C & $\%$ & No. C & $\%$ & No. C & $\%$ \\
\hline No complications & 61 & 35.88 & 135 & 37.92 & 79 & 37.09 & 55 & 38.19 & 330 & 37.37 \\
\hline $\begin{array}{l}\text { Menstrual } \\
\text { irregularities }\end{array}$ & 12 & 7.06 & 27 & 7.58 & 16 & 7.51 & 11 & 7.64 & 66 & 7.47 \\
\hline White discharge & 24 & 14.12 & 52 & 14.61 & 31 & 14.55 & 21 & 14.58 & 128 & 14.50 \\
\hline Low back ache & 23 & 13.53 & 50 & 14.04 & 29 & 13.62 & 20 & 13.89 & 122 & 13.82 \\
\hline $\begin{array}{l}\text { Lower abdominal } \\
\text { pain }\end{array}$ & 7 & 4.12 & 18 & 5.06 & 10 & 4.69 & 7 & 4.86 & 42 & 4.76 \\
\hline General weakness & 9 & 5.29 & 19 & 5.34 & 12 & 5.63 & 8 & 5.56 & 52 & 5.89 \\
\hline Easy fatigability & 10 & 5.88 & 21 & 5.90 & 13 & 6.10 & 8 & 5.56 & 48 & 5.44 \\
\hline PID & 2 & 1.18 & 1 & 0.28 & 0 & 0.00 & 0 & 0.00 & 1 & 0.11 \\
\hline Ectopic & 0 & 0.00 & 1 & 0.28 & 0 & 0.00 & 0 & 0.00 & 4 & 0.45 \\
\hline Dyspareunia & 4 & 2.35 & 7 & 1.97 & 8 & 3.76 & 1 & 0.69 & 20 & 2.27 \\
\hline Weight gain & 9 & 5.29 & 6 & 1.69 & 3 & 1.41 & 1 & 0.69 & 19 & 2.15 \\
\hline Weight loss & 2 & 1.18 & 4 & 1.12 & 2 & 0.94 & 6 & 4.17 & 14 & 1.59 \\
\hline Scar Tenderness & 0 & 0.00 & 0 & 0.00 & 0 & 0.00 & 0 & 0.00 & 0 & 0.00 \\
\hline Miscellaneous & 7 & 4.12 & 15 & 4.21 & 9 & 4.23 & 6 & 4.17 & 37 & 4.19 \\
\hline $\begin{array}{l}\text { Total No. of Cases } \\
\& \%\end{array}$ & 170 & 100 & 356 & 100 & 213 & 100 & 144 & 100 & 83 & 100 \\
\hline Chi-Square $=26.096$ & $\mathrm{lf}=39$ & & & & & & & & & \\
\hline
\end{tabular}

The complications were more noticed in housewives when compared to working women in both groups (Table 12 and 13).

With reference to Table 14 and 15, the complications in both acceptors were more in low socio economic status group. The leading complications in higher socioeconomic groups were PID and dyspareunia.

As evident from table 16, the overall menstrual irregularities were $16 \%$ in both groups. This was more in conventional tubectomy group $(18.8 \%)$ compared to $13.2 \%$ in DPL group. Overall occurrence of lactational amenorrhoea was $3.1 \%$ which was more in conventional tubectomy acceptors $(3.6 \%)$ than that of $2.6 \%$ in DPL group. The leading menstrual disorder was menorrhagia in both acceptors with overall rate of $4.2 \%$. Menorrhagia was more common in conventional tubectomy acceptors (4.6\%) compared to $3.6 \%$ of DPL group. The second leading menstrual disorder was dysmenorrhoea in both acceptors with overall rate of $3.4 \%$. It was more in conventional tubectomy acceptors $(3.2 \%)$ that of $3.2 \%$ in DPL group. The other menstrual disorders were irregular periods, polymenorrhoea, oligomenorrhoea and scanty menstruation and these were more in conventional tubectomy acceptors compared with DPL group. 
Table 12: Complications of CTL group with relation to occupation.

\begin{tabular}{|c|c|c|c|c|c|c|}
\hline \multirow[t]{2}{*}{ Complications } & \multicolumn{2}{|c|}{ House Wife } & \multicolumn{2}{|c|}{ Working Women } & \multicolumn{2}{|l|}{ Total } \\
\hline & $\begin{array}{l}\text { No. of } \\
\text { Cases }\end{array}$ & $\%$ & $\begin{array}{l}\text { No. of } \\
\text { Cases }\end{array}$ & $\%$ & $\begin{array}{l}\text { No. of } \\
\text { Cases }\end{array}$ & $\%$ \\
\hline No complications & 173 & 27.37 & 102 & 26.70 & 275 & 27.12 \\
\hline Menstrual irregularities & 59 & 9.34 & 35 & 9.16 & 94 & 9.27 \\
\hline White discharge & 79 & 12.50 & 47 & 12.30 & 126 & 12.43 \\
\hline Low back ache & 105 & 16.61 & 62 & 16.23 & 167 & 16.47 \\
\hline Lower abdominal pain & 45 & 7.12 & 27 & 7.07 & 72 & 7.10 \\
\hline General weakness & 48 & 7.59 & 28 & 7.33 & 76 & 7.50 \\
\hline Easy Fatigability & 40 & 6.33 & 23 & 6.02 & 63 & 6.21 \\
\hline PID & 2 & 0.32 & 4 & 1.05 & 6 & 0.59 \\
\hline Ectopic & 1 & 0.16 & 0 & 0.00 & 1 & 0.10 \\
\hline Dyspareunia & 15 & 2.37 & 14 & 3.66 & 29 & 2.86 \\
\hline Weight gain & 13 & 2.06 & 10 & 2.62 & 23 & 2.27 \\
\hline Weight loss & 10 & 1.58 & 6 & 1.57 & 16 & 1.58 \\
\hline Scar Tenderness & 6 & 0.95 & 3 & 0.79 & 9 & 0.89 \\
\hline Miscellaneous & 36 & 5.70 & 21 & 5.50 & 57 & 5.62 \\
\hline Total No. of Cases \& \% & 632 & 100 & 682 & 100 & 1014 & 100 \\
\hline Chi-Square $=4.696, \mathrm{df}=13$ & & & & & & \\
\hline
\end{tabular}

Table 13: Complications of DPL group with relation to occupation.

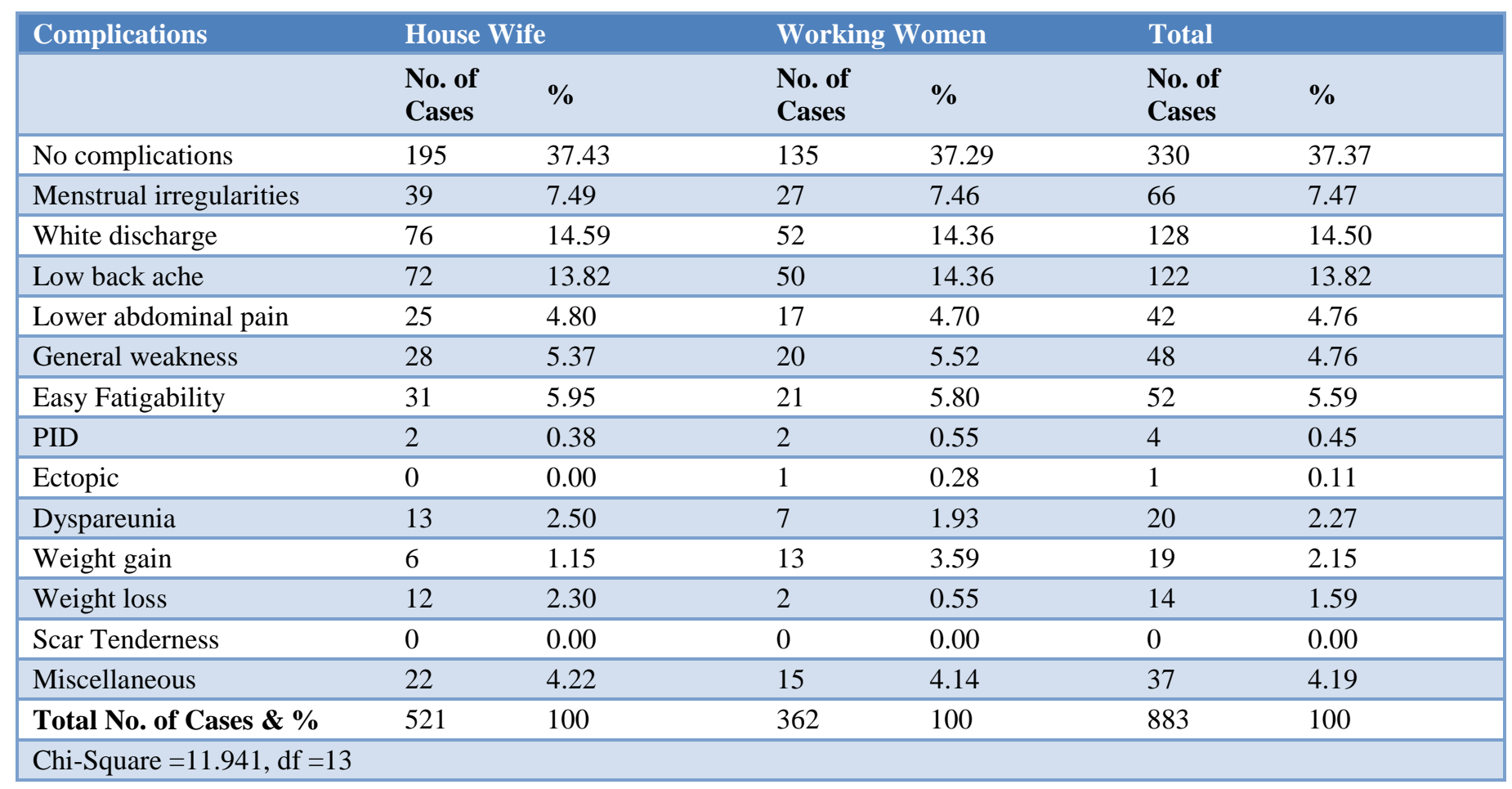


Table 14: Complications of CTL based on economic status.

\begin{tabular}{|c|c|c|c|c|c|c|c|c|}
\hline Complications & $<1200$ & & 12000 & $000 \mathrm{RS}$ & $>36000$ & & Total & \\
\hline & $\begin{array}{l}\text { No. of } \\
\text { Cases }\end{array}$ & $\%$ & $\begin{array}{l}\text { No. of } \\
\text { Cases }\end{array}$ & $\%$ & $\begin{array}{l}\text { No. of } \\
\text { Cases }\end{array}$ & $\%$ & $\begin{array}{l}\text { No. of } \\
\text { Cases }\end{array}$ & $\%$ \\
\hline No complications & 149 & 27.39 & 96 & 27.43 & 30 & 25.00 & 275 & 27.12 \\
\hline Menstrual irregularities & 51 & 9.38 & 33 & 9.43 & 10 & 8.33 & 94 & 9.27 \\
\hline White discharge & 69 & 12.68 & 44 & 12.57 & 13 & 10.83 & 126 & 12.43 \\
\hline Low back ache & 91 & 16.73 & 58 & 16.57 & 18 & 15.00 & 167 & 16.47 \\
\hline Lower abdominal pain & 39 & 7.17 & 25 & 7.14 & 8 & 6.67 & 72 & 7.10 \\
\hline General weakness & 41 & 7.54 & 26 & 7.43 & 9 & 7.50 & 76 & 7.50 \\
\hline Easy Fatigability & 34 & 6.25 & 22 & 6.29 & 7 & 5.83 & 63 & 6.21 \\
\hline PID & 3 & 0.55 & 2 & 0.57 & 1 & 0.83 & 6 & 0.59 \\
\hline Ectopic & 0 & 0.00 & 1 & 0.29 & 0 & 0.00 & 1 & 0.10 \\
\hline Dyspareunia & 16 & 2.94 & 9 & 2.57 & 4 & 3.333 & 29 & 2.86 \\
\hline Weight gain & 13 & 2.39 & 7 & 2.00 & 3 & 2.50 & 23 & 2.27 \\
\hline Weight loss & 3 & 0.55 & 4 & 1.14 & 9 & 7.50 & 16 & 1.58 \\
\hline Scar Tenderness & 4 & 0.74 & 3 & 0.86 & 2 & 1.67 & 9 & 0.89 \\
\hline Miscellaneous & 31 & 5.70 & 20 & 5.71 & 6 & 5.00 & 57 & 5.62 \\
\hline $\begin{array}{l}\text { Total No. of Cases \& } \\
\%\end{array}$ & 544 & 100 & 350 & 100 & 120 & 100 & 1014 & 100 \\
\hline
\end{tabular}

Table 15: Complications of DPL based on economic status.

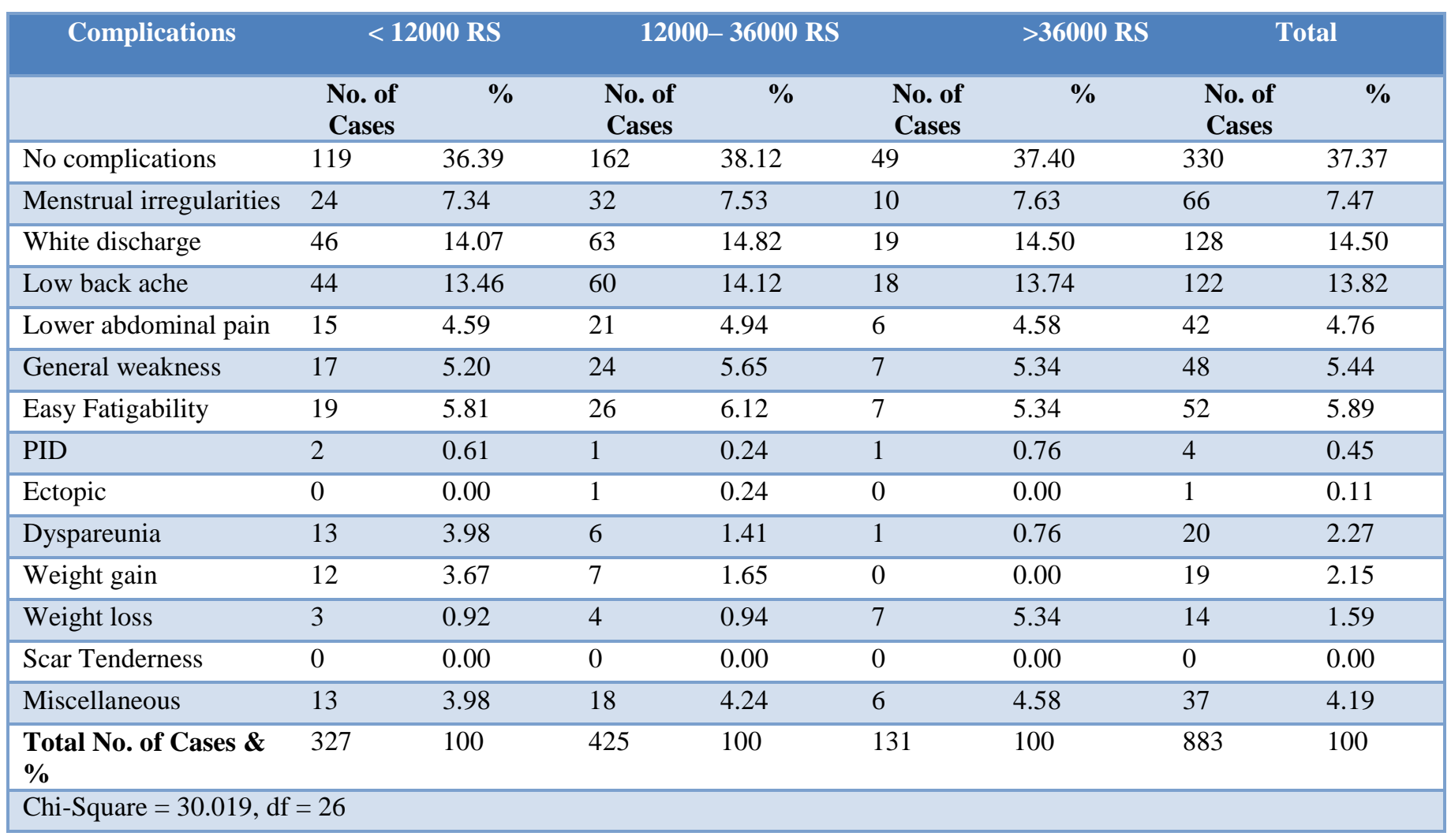


Table 16: Menstrual characteristics in both groups.

\begin{tabular}{|lllllll|}
\hline & DPL & \multicolumn{2}{l}{ Con Tubectomy } & \multicolumn{2}{l|}{ Total } \\
& $\begin{array}{l}\text { No. of } \\
\text { Cases }\end{array}$ & $\mathbf{\%}$ & $\begin{array}{l}\text { No. of } \\
\text { Cases }\end{array}$ & $\%$ & No. of Cases & \% \\
\hline Lactational Amenorrhea & 13 & 2.6 & 18 & 3.6 & 31 & 3.1 \\
\hline Menorrhagia & 18 & 3.6 & 24 & 4.8 & 42 & 4.2 \\
\hline Irregular Periods & 10 & 2.0 & 17 & 3.4 & 27 & 2.7 \\
\hline Polymenorrhoea & 3 & 0.6 & 7 & 1.4 & 10 & 1.0 \\
\hline Oligo Menorrhoea & 4 & 0.8 & 6 & 1.2 & 10 & 1.0 \\
\hline Scanty Menstruation & 2 & 0.4 & 4 & 0.8 & 6 & 0.6 \\
\hline Dysmenorrhoea & 16 & 3.2 & 18 & 3.6 & 34 & 3.4 \\
\hline No complaints & 434 & 86.8 & 406 & 81.2 & 840 & 84 \\
\hline Total No. of Cases \& \% & $66(13.2 \%)$ & 100 & $94(18.8 \%)$ & 100 & $160(16 \%)$ & 100 \\
\hline Chi-Square = 1.406, df = 6 & & & & & & \\
\hline
\end{tabular}

\section{DISCUSSION}

In this study 1000 acceptors were divided into two groups: DPL acceptors \& CTL acceptors. Various factors like age, literacy, occupation, socioeconomic status were evaluated and compared with the complications.

Maximum numbers of acceptors $(82.2 \%)$ were between 21-30 years of age. The average age at which the acceptors were operated in $24.1 \%$, in DPL group (24.4\%) and the conventional tubectomy group was $23.8 \%$. Acceptance of sterilization among Hindus was $87.7 \%$ against their population $82 \%$. The Muslim acceptors were $7.5 \%$ against their population $13 \%$, whereas Christian acceptors were $4.8 \%$ against their population of $5 \%$. It shows Hindus fare better in terms of acceptance compared to Muslims \& Christians. Illiterates and acceptors having education below primary education constitute $69 \%$ of total acceptors showing that literacy status did not influence much on the acceptance of tubectomy. There was slight preponderance of DPL acceptance in high literacy group. Previous studies have demonstrated that the mortality rate for the procedures ranged from $0.40-16 \% .{ }^{7,8}$ However this study did not exhibit mortality and showed that complications were more in conventional tubectomy group compared to DPL group.

Williams et $\mathrm{al}^{6}$ have demonstrated that there are a higher frequency of gynecological diseases in a sterilized population than expected in the general female population. In their series of 200 patients, $24 \%$ developed clinically significant gynecologic disorders following the operation. According to Haynes DM \& Wolfe $\mathrm{WM}^{9}$, incidence of gynaecologic problems were $31 \%$ in tubally sterilized women.

The overall incidence of post ligation menorrhagia was $42 \%$ (Stock RJ, 1978) (10 $^{10}$ and "post tubal ligation syndrome" variably characterized by pelvic discomfort, ovarian cyst formation and menorrhagia which were assumed to happen owing to uterovarian circulatory disturbance (Williams, 1951). ${ }^{6}$

Post-tubal ligation syndrome includes pain during intercourse, aching lower back, premenstrual tension syndrome, difficulty in menstruating, uterine hemorrhage, and absence of menstruation. The syndrome is caused by blood circulation problems in and around the Fallopian tubes and ovaries, pressure on nerves, and intrapelvic adhesion. ${ }^{11}$

Our study showed that delayed complications like low back ache, menstrual disturbances, scan tenderness, lower abdominal pain etc were more in CTL group when to compared to DPL group. The various menstrual disturbances were more in conventional tubectomy group compared to DPL group. Psychological upsets of minor nature are present in $2.5 \%$ of cases in the study. Even though there were delayed complications in both groups, the results were not significant. The present study clearly revealed that age, religion, literacy \& socioeconomic status do not have a major role in occurrence of complications and symptoms of post ligation syndrome doesn't exist in both groups. 


\section{CONCLUSIONS}

Age, religion, literacy status, economic status \& occupation of acceptors did not influence much the occurrence of complications. Post tubal ligation syndrome does exist in both groups. Nevertheless, much more population studies are needed in order to confirm this finding.

\section{ACKNOWLEDGEMENTS}

We sincerely thank all women who gave consent and participated in this study.

\section{Funding: No funding sources}

Competing interests: There are no competing interests to declare

Ethical approval: The study was approved by the Institutional ethics committee

\section{REFERENCES}

1. EngenderHealth; Contraceptive Sterilization Global Issues and Trends. Sterilization Incidence and Prevalence. EngenderHealth; 2002:139-58.

2. Pati S, Cullins V. Female sterilization. Evidence. Obstet Gynecol Clin North Am 2000;27:859-99.

3. Stewart GK, Carignan CS. Female and male sterilization. In Contraceptive technology, 17th revised ed. Hatcher RA et al, eds. New York: Ardent Media; 1998:545-88.

4. WHO 1992. Female sterilization: A guide to provision of services. Geneva.

5. Peterson HB, Jeng G, Folger SG, Hillis SA, Marchbanks PA, Wilcox LS. The risk of menstrual abnormalities after tubal sterilization. U.S. Collaborative Review of Sterilization Working Group. N Engl J Med 2000;343:1681-7.

6. Williams El, Jones HE, Merrill RE, The subsequent course of patients sterilized by tubal ligation. Am J Obstet Gynecol 1951;61:423-6.

7. Sitompul H, Lun KC, Lumbanraja M, Kabran RM, Albar E, Simanjuntak P, Hanafiah MJ. Comparison of three types of tubal sterilization: the Medan experience. Contraception 1984;29:55-63.

8. Taner CE, Aban M, Yilmaz N, Senturk N, Toy E. Pomeroy tubal ligation by laparoscopy and minilaparotomy. Adv Contracept 1994;10:151-5.

9. Haynes DM, Wolfe WM. Tubal sterilization in an indigent population. Report of fourteen years' experience. Am J Obstet Gynecol 1970;106:104453.

10. Stock RJ. Evaluation of sequelae of tubal ligation. Fertil Steril 1978;29:169-74.

11. Satoh K, Osada H. Post-tubal ligation syndrome. Ryoikibetsu Shokogun Shirizu 1993;(1):772-3.

DOI: $10.5455 / 2320-1770 . i j r \operatorname{cog} 20130201$

Cite this article as: Palli SB, Akkupalli VL. A study to evaluate the relationship between socioeconomic, demographic characteristics and complications of acceptors in double puncture laparoscopic and conventional tubal ligation procedures. Int J Reprod Contracept Obstet Gynecol 2013;2:1-11. 